\title{
Reduced CD73 expression and its association with altered purine nucleotide metabolism in colorectal cancer cells robustly causing liver metastases
}

\author{
MASAHIRO MATSUYAMA ${ }^{1,3}$, MASATOSHI WAKUI ${ }^{3,5,6}$, MAKOTO MONNAI $^{3,4}$, TOMOKO MIZUSHIMA $^{3}$, \\ CHIYOKO NISHIME ${ }^{3,5}$, KENJI KAWAI ${ }^{3,5}$, MITSUYO OHMURA ${ }^{5}$, HIROSHI SUEMIZU ${ }^{3}$, TAKAKO HISHIKI ${ }^{5}$, \\ MAKOTO SUEMATSU ${ }^{5}$, MITSURU MURATA ${ }^{6}$, TSUYOSHI CHIJIWA ${ }^{2,7}$, DAISUKE FURUKAWA ${ }^{1,3}$, \\ KYOJI OGOSHI ${ }^{1}$, HIROYASU MAKUUCHI $^{1}$ and MASATO NAKAMURA ${ }^{2,3}$
}

\begin{abstract}
Departments of ${ }^{1}$ Surgery, and ${ }^{2}$ Pathology, Tokai University School of Medicine, Isehara; ${ }^{3}$ Central Institute for Experimental Animals (CIEA), Nogawa, Miyamae-ku, Kawasaki; ${ }^{4}$ Chugai Research Institute for Medical Science, Inc., Kajiwara, Kamakura, Kanagawa; Departments of ${ }^{5}$ Biochemistry and Integrative Medical Biology, and ${ }^{6}$ Laboratory Medicine, School of Medicine, Keio University Shinjuku-ku; ${ }^{7}$ Disaster Medical Center, Tachikawa, Tokyo, Japan
\end{abstract}

Received November 20, 2009; Accepted February 26, 2010

DOI: 10.3892/ol_00000076

\begin{abstract}
Liver metastases of colorectal cancers significantly affect the prognoses of patients. To further understand the biological aspects of the metastatic phenotypes, we established the highly liver-metastatic human colorectal cancer cell subline SW48LM2. The subline was established through the serial intrasplenic transfer of cells derived from poor but visible hepatic tumor foci formed by parental SW48 cells and transferred to NOD/SCID/IL-2R $\gamma \mathrm{c}^{\text {null }}$ mice. The growth, both under monolayer culture conditions and during the formation of subcutaneous tumors, was similar between the two cell lines, although there were morphological differences in the in vitro spheroid formation. Of 41 molecules reportedly associated positively or negatively with tumor progression, four were overexpressed and four were underexpressed in SW48LM2 cells. Notably, this liver-metastatic cell subline exhibited a strongly reduced expression of the ecto-5'nucleotidase CD73 as well as an altered metabolism of purine nucleotides. Previous studies showed a positive correlation between CD73 expression and metastatic cancer phenotypes. A reduced CD73 expression in tumor cells, however, may contribute to obtaining insight into the mechanisms of liver metastases.
\end{abstract}

Correspondence to: Dr Masatoshi Wakui, Department of Laboratory Medicine, School of Medicine, Keio University, 35 Shinanomachi, Shinjuku-ku, Tokyo 160-8582, Japan

E-mail: mwakui@sc.itc.keio.ac.jp

Key words: CD73, colorectal cancer, liver metastasis

\section{Introduction}

Liver metastases are frequently inoperative and significantly affect the prognoses of patients with cancer. To develop novel strategies for early diagnostics and curative therapies, a further understanding of tumor cell adaptation to the hepatic microenvironment is required. NOD/SCID/IL-2R $\gamma \mathrm{c}^{\text {null }}(\mathrm{NOG})$ mice exhibit strongly immunodeficient phenotypes due to the lack of T, B and NK cells and impairments in certain innate immune responses, allowing for the construction of liver metastasis models with stable reproducibility of the experimental outcome $(1,2)$. Through liver metastasis models that employed NOG mice, a highly liver-metastatic human pancreatic cancer cell subline, derived from a poorly liver-metastatic parental line, was previously established and examined. Moreover, a key molecular regulator of liver metastasis was identified (2).

Colorectal cancer types commonly metastasize to the liver although some patients present operable liver metastases, in contrast to patients with pancreatic cancer types (3). The similarities or differences in the biology of liver metastases between colorectal and pancreatic cancer types remain to be elucidated. The present study established and examined a highly liver-metastatic human colorectal cancer cell subline through experimental procedures analogous to our previous study (2). Notably, the ecto-5'-nucleotidase CD73 (also known as NT5E), which catalyzes the conversion of purine $5^{\prime}$ mononucleotides to nucleosides, was poorly expressed in the established SW48LM2 cell subline compared to the parental SW48 cell line. Consistent with a reduced CD73 expression, results of the metabolomic analysis showed an altered metabolism of purine nucleotides. In contrast to a number of studies showing a positive association of CD73 expression with metastatic phenotypes, these results suggest that a reduction in CD73 expression in tumor cells is involved in the mechanisms by which liver metastases are formed. 


\section{Materials and methods}

Cells. The human colorectal cancer cell line SW48 was purchased from the American Type Culture Collection (Manassas, VA, USA). The SW48LM2 cell subline was established as described below. SW48 and SW48LM2 were maintained in Leibovitz's L-15 (Sigma) supplemented with antibiotics and $10 \%$ fetal bovine serum (HyClone, UT, USA). Prior to being transferred to the mice, the cell lines were incubated in a $100 \%$ air $\left(37^{\circ} \mathrm{C}\right)$ incubator and passaged on reaching $80 \%$ confluence. To culture SW48 and SW48LM2 cells for 7 days under hypoxia, the AnaeroPack Anaero cultivation system with AnaeroPack MicroAero (Mitsubishi Gas Chemical, Japan) was used, providing 8-9\% $\mathrm{O}_{2}$ conditions.

Establishment of a highly liver-metastatic cell subline from the parental SW48. The in vivo experiments were performed in accordance with institutional guidelines and were approved by the Animal Experimentation Committee of the Tokai University, the Keio University and the Central Institute for Experimental Animals (CIEA). NOG mice were bred and used at the age of 7-9 weeks. Liver metastases were experimentally induced by intrasplenic transfer of human cancer cells as described in a previous study (4). NOG mice were euthanized 6 weeks after the intrasplenic transfer of $1 \times 10^{5}$ SW48 cells, which metastasize poorly to the liver (5). The livers were removed from the mice and macroscopically observed. Hepatic metastatic foci with diameters $>2 \mathrm{~mm}$ were collected and cut into $1-\mathrm{mm}^{3}$ cubes. Foci were dispersed into single cells in a solution of trypsin-EDTA (IBL Co., Ltd., Japan). Contamination with mouse-derived cells was examined by PCR using the primers: forward, 5'-TGTAG GTACTAACACTGGCTCGTGTGACAA-3' and reverse, 5'-GGTGTTGAAGGTCTCAAACATGATCTGTA-3'. PCR with this set of primers can amplify both human and mouse $\beta$-actin genes as 247- and 273-bp DNA fragments, respectively. To examine for contamination with other human cell lines, short tandem repeat analysis was carried out using the markers: D5S818, D13S317, D7S820 and DS16S539. Primer information is available at the UniSTS database on the NCBI website (http://www.ncbi.nlm.njh.gov/). To confirm the ability to form liver metastases, the cells derived from the metastatic foci, i.e., SW48LM2 cells, were cultured and intrasplenically transferred at $1 \times 10^{4}$ cells to a new cohort of NOG mice, followed by macroscopic observation of livers removed 6 weeks after the transfer.

Subcutaneous tumor formation. For subcutaneous tumor formation, $1 \times 10^{4}$ SW48 or SW48LM2 cells suspended in $0.2 \mathrm{ml}$ of serum-free medium were subcutaneously transferred to NOG mice. Palpable tumors were measured weekly, and the mice were sacrificed for histopathological examination 6 weeks after the transfer.

Evaluation of morphological invasiveness of subcutaneous tumors by fractal dimension analysis. For quantitative evaluation of the invasiveness based on the geometry of tumor tissues, fractal dimension analysis was applied to the histological findings as reported in a previous study (6). Mice were sacrificed 2 weeks after the subcutaneous transfer, followed by harvesting of the formed tumors. Paraffin-embedded samples were sectioned at $5 \mu \mathrm{m}$. Immunostaining of the sections with the mouse monoclonal anti-multi-cytokeratin (clone AE1/ AE3; Leica Microsystems, Japan) was performed on the Bond Max system (Leica Microsystems). The photographic images of the immunostained sections were prepared by the Imager M1 (Carl Zeiss Microimaging, Japan) and then converted to the gray-scaled images using Photoshop CS3 (Adobe Systems Inc., San Jose, CA, USA). The fractal dimension analysis was carried out using the software ImageJ version 1.33 (http://rsb. info.nih.gov/ij/).

Assay of in vitro invasion of cancer cells. Comparison of the invasiveness of SW48 and SW48LM2 cells in vitro was carried out using the BD BioCoat Matrigel invasion chamber (BD Biosciences, Bedford, MA, USA) as recommended by the manufacturer.

Assay of spheroid formation of cancer cells. The spheroid culture was carried out using the Sumilon Celltight Spheroid 96U (Sumitomo Bakelite, Japan). The viable cell numbers were analyzed using the Cell Counting Kit-8 (Dojindo Laboratories, Japan) as recommended by the manufacturer.

Real-time RT-PCR assay. Total RNA was extracted from collected cells using the TRIzol reagent (Invitrogen, Carlsbad, CA, USA) and subjected to cDNA synthesis with the PrimeScript RT reagent kit (Takara Bio Inc., Japan). The reactions were prepared with the obtained cDNA and the SYBR Premix Ex Taq II. Gene-specific primers were designed by the Perfect Real Time support system (Takara Bio Inc.) and subjected to the real-time PCR assay using the Thermal Cycler Dice Real Time system (Takara Bio Inc.). The examined molecules were: VEGF-A, VEGF189, TSP1, TSP2, MMP2, MMP7, E-cadherin, S100A4, CA9, CA12, HIF1a, HIF2a, NME1, GLUT1, GLUT3, CYGB, MYC, MCT4, SCO2, COX4-1, COX4-2, TLR3, TLR4, TLR5, TLR7, TLR9, MCL1, LDHA, LDHB, ALDH1L2, ALDH3A1, TFAM, UCP2, TWIST, Slug, Snail, ZEB1, ZEB2, vimentin, CD73, CD47, NT5M, NT5C1A, NT5C1B and NT5C2.

Metabolomic analysis using capillary electrophoresismass spectrometry (CE-MS). Extracts from the pellets of cultured cells were measured by the Agilent CE Capillary Electrophoresis system equipped with an air pressure pump, an Agilent 1100 series MSD mass spectrometer and an Agilent 1100 series isocratic high performance liquid chromatography pump, a G1603A Agilent CE-MS adaptor kit and G1607A Agilent CE-MS sprayer kit (Agilent Technologies) as previously described (7).

Flow cytometric analysis. Cells were stained with PE conjugated mouse anti-human CD73 (clone AD2; BD Biosciences, San Diego, CA, USA). The flow cytometric analysis was performed using MoFlo (Beckman Coulter, Miami, FL, USA).

Statistical analysis. Statistical analysis was carried out using the two-sample t-test or Fisher's exact test. Values of $p<0.05$ were considered to be statistically significant. 
A
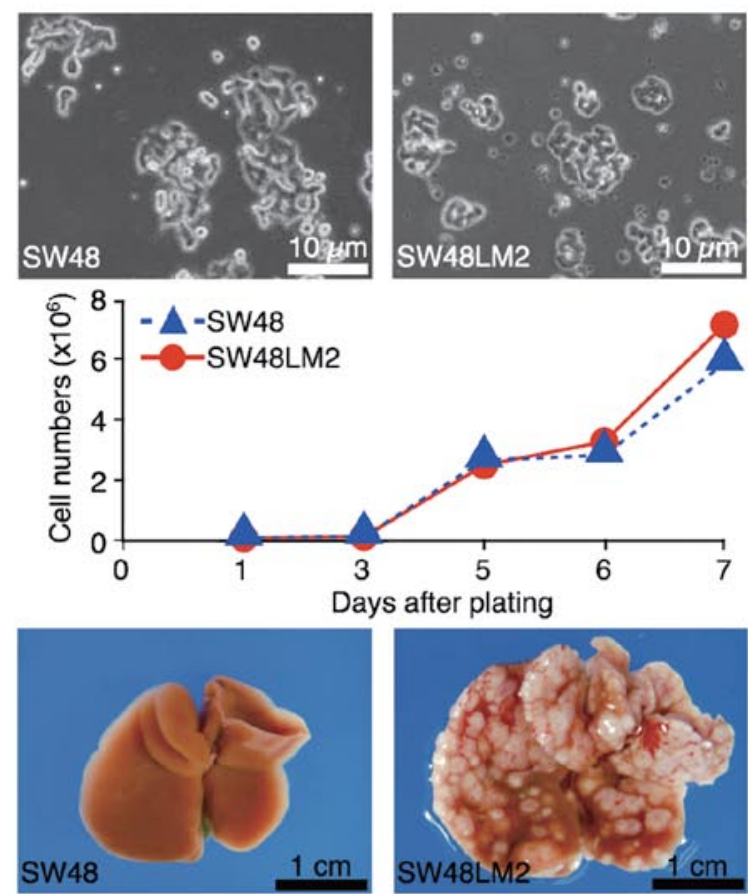

B
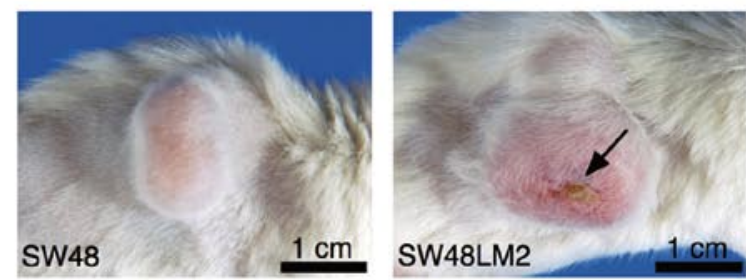

\begin{tabular}{lcc}
\hline & SW48 $(n=6)$ & SW48LM2 $(n=6)$ \\
\hline Thoracic involvement & $1 / 6$ & $3 / 6$ \\
Ulceration & $0 / 6$ & $3 / 6$ \\
Liver Metastasis & $0 / 6$ & $0 / 6$ \\
\hline
\end{tabular}

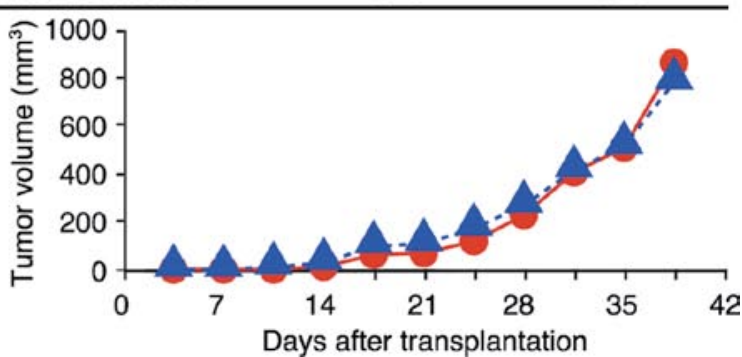

Figure 1. (A) Establishment of the highly liver-metastatic cell line, SW48LM2, from the poorly liver-metastatic parental line SW48. The cell morphology and growth under monolayer culture conditions were similar between the two lines, although intrasplenically transferred SW48LM2 cells rapidly formed hepatic metastatic foci in NOG mouse recipients. (B) Comparison of subcutaneous tumors formed by SW48 and SW48LM2 cells. The tumor sizes were similar throughout the time period.

\section{Results}

Establishment of a highly liver-metastatic human colorectal cancer cell subline. Poorly formed metastatic foci were noted in the liver following the intrasplenic transfer of SW48 cells to NOG mice (Fig. 1A). Cells were isolated from the visible foci and were then subjected to the short-term culture. The intrasplenic transfer of the cultured cells to NOG mice led to the rapid formation of hepatic metastatic foci (Fig. 1A). This established cell subline was termed SW48LM2. The growth
A

SW48 Subcutaneous tumor

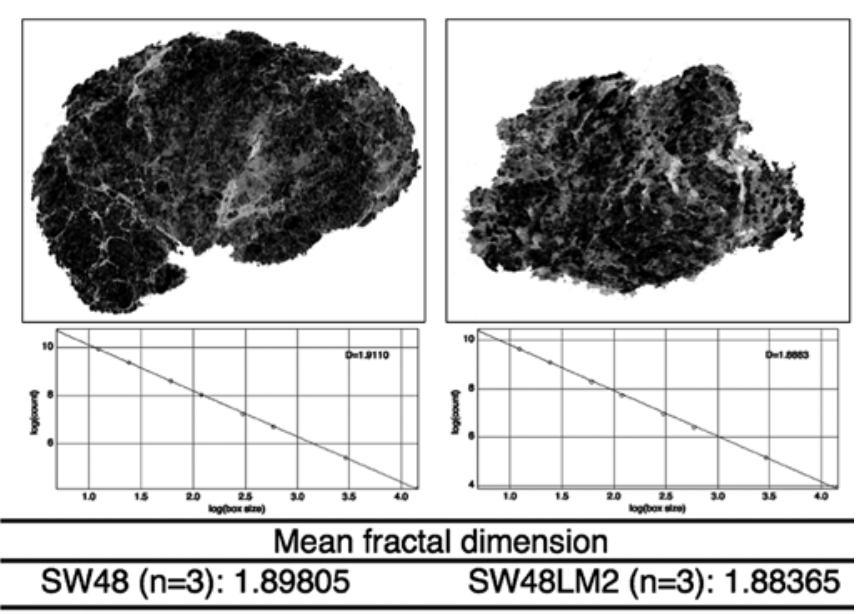

B Spheroid culture
SW48

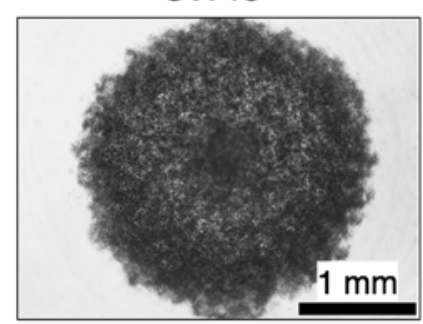

\section{Spheroid culture SW48LM2}
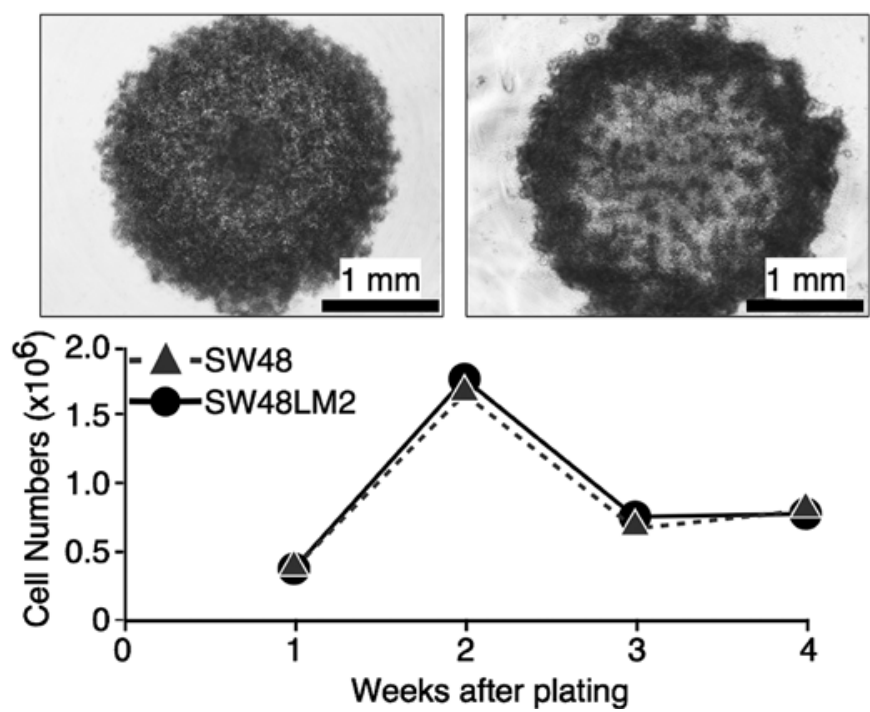

Figure 2. (A) Morphologic evaluation of the invasiveness of subcutaneous tumors. The fractal dimension analysis showed a similarity in the morphologic invasiveness of the subcutaneous tumors of SW48 and SW48LM2 cells. (B) In vitro spheroid formation. The spheroids formed by SW48LM2 cells were morphologically different from those formed by SW48 cells, although the viable cell numbers were similar throughout the time period.

under monolayer culture conditions was similar between the SW48 and SW48LM2 cells (Fig. 1A).

Comparison of subcutaneous tumor formation and in vitro spheroid formation in the SW48 and SW48LM2 cells. The growth features of the subcutaneous tumors were similar between the SW48 and SW48LM2 cells (Fig. 1B). In the subcutaneous transfer, no metastases were macroscopically observed in the liver or other organs, except thoracic involvement and skin ulcerations. The latter metastases tended to occur more frequently in the subcutaneous transfer of SW48LM2 cells, although the differences were not statistically significant (Fig. 1B). For evaluation of the invasiveness of the SW48LM2 cells based on tumor shape profiles, subcutaneous tumors, formed 2 weeks after the transfer, were 
CD73

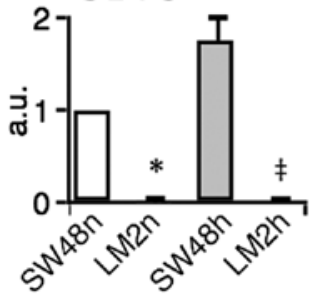

ALDH1L2

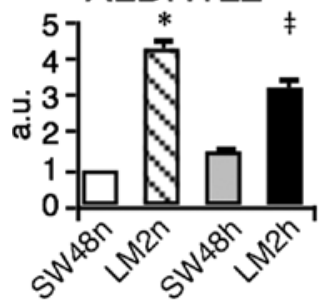

VEGF189

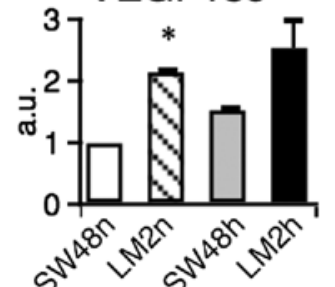

MCT4

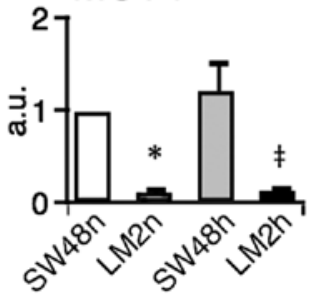

ALDH3A1

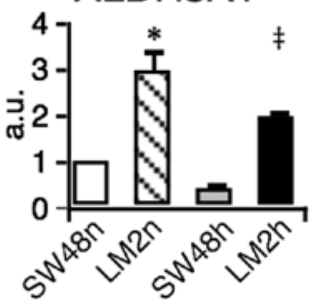

TLR3

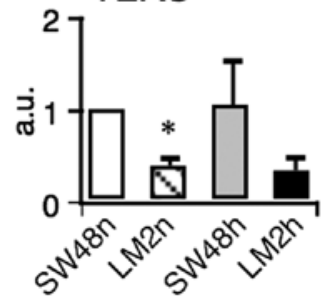

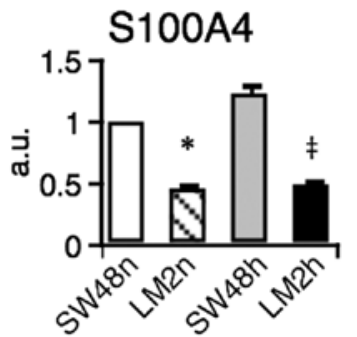

Vimentin

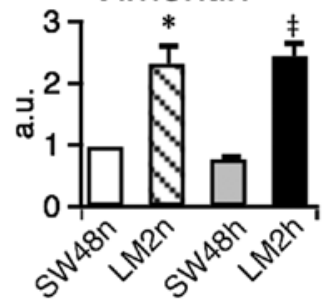

SW48n : SW48 normoxia

LM2n : SW48LM2 normoxia

SW48h : SW48 hypoxia

LM2h : SW48LM2 hypoxia

${ }^{*} \mathrm{P}<0.05$ when compared with SW48 under normoxia

${ }_{P}<0.05$ when compared with SW48 under hypoxia

Figure 3. Differential gene expression in SW48 and SW48LM2 cells cultured under normoxia or hypoxia. Four overexpressed and four underexpressed genes were detected in SW48LM2 cells. The differences between the two cell lines were statistically significant $(\mathrm{p}<0.05)$, except for differences in the levels of the TLR3 gene transcript and VEGF189 splicing form transcripts under hypoxia.

subjected to fractal dimension analysis. The mean fractal dimensions showed that the two cell lines were similar in morphological invasiveness (Fig. 2A). In addition, the in vitro invasion assay detected no significant differences between SW48 and SW48LM2 cells (data not shown). Similar changes in the viable cell numbers were observed throughout the time period of the in vitro spheroid formation between the SW48 and SW48LM2 cells, whereas the spheroids were morphologically different (Fig. 2B). As internal hypoxia acutely affects spheroid formation via tumor cells, such morphological differences appeared to reflect the distinct features of the cells with respect to hypoxic adaptation (8). We therefore carried out the analysis of monolayer-cultured cells under normoxia and hypoxia.

Profiles of gene expression potentially associated with tumor progression in the SW48 and SW48LM2 cells. Gene transcripts of 41 molecules reportedly associated positively or negatively with tumor growth, invasion or metastases were analyzed by real-time RT-PCR (2,9-16). Four overexpressed and four underexpressed genes were detected in the SW48LM2 cells (Fig. 3). Notably, an $~ 50$-fold reduction under normoxia and an $\sim 100$-fold reduction under hypoxia were observed with respect to the ecto-5'-nucleotidase CD73 (also known as NT5E) gene transcripts. Of note was that the gene transcripts of CD73, the monocarboxylate transporter MCT4, and S100A4 were reduced in the liver-metastatic cell line, conflicting with previous reports showing a positive association with metastatic cancer phenotypes (2,9-12). In contrast, a lower expression of the TLR3 gene as well as a higher expression of aldehyde dehydrogenase ALDH1L2 and ALDH3A1 genes and a splicing variant of VEGF-A, VEGF189, corroborated reported findings (13-15). It appeared plausible that an epithelial mesenchymal transition-associated molecule, vimentin, was highly expressed in the SW48LM2 cells (16). Differential expression of genes encoding metabolic enzymes and transporter prompted us to examine the metabolomic profiles of the SW48 and SW48LM2 cells.

Profiles of metabolites in the SW48 and SW48LM2 cells. Metabolites of glycolysis, the pentose phosphate pathway, TCA cycle and anabolic and catabolic reactions of amino acids, as well as nucleotides in the SW48 and SW48LM2 cells were analyzed by CE-MS. As a result, a decrease in purine but not pyrimidine nucleosides and a reciprocal increase in purine nucleotides were observed in the SW48LM2 cells under normoxia and hypoxia, suggesting a possible association with a reduced CD73 expression (Fig. 4A).

Expression of nucleotidases converting purine nucleotides to nucleosides in the SW48 and SW48LM2 cells. To rule out the contribution of four purine nucleotidases other than CD73 to the altered metabolism, the expression levels of the nucleotisades were analyzed by real-time RT-PCR (Fig. 4B). No significant 
A

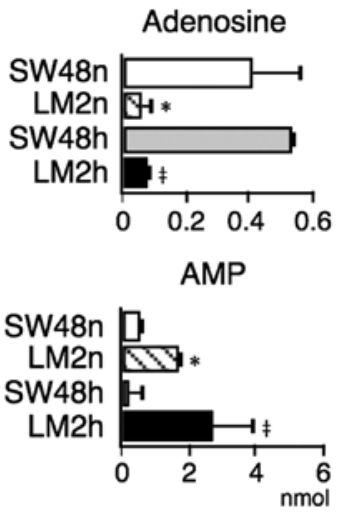

Thymidine

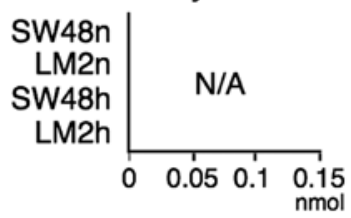

TMP

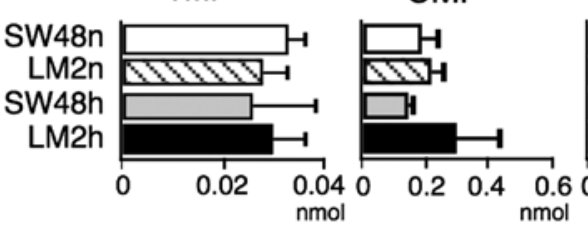

SW48n: SW48 normoxia, LM2n: SW48LM2 normoxia, SW48h: SW48 hypoxia, LM2h: SW48LM2 hypoxia

${ }^{*} \mathrm{P}<0.05$ when compared with SW48 under normoxia $\ddagger_{\mathrm{P}}<0.05$ when compared with SW48 under hypoxia

B
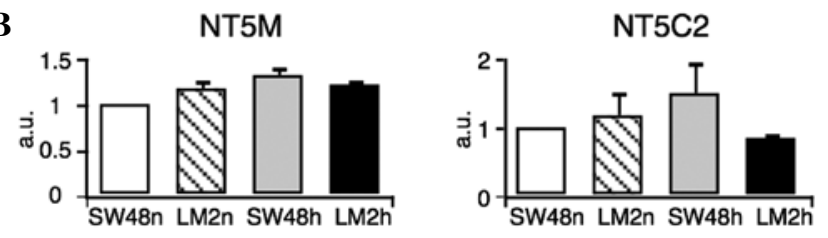

C

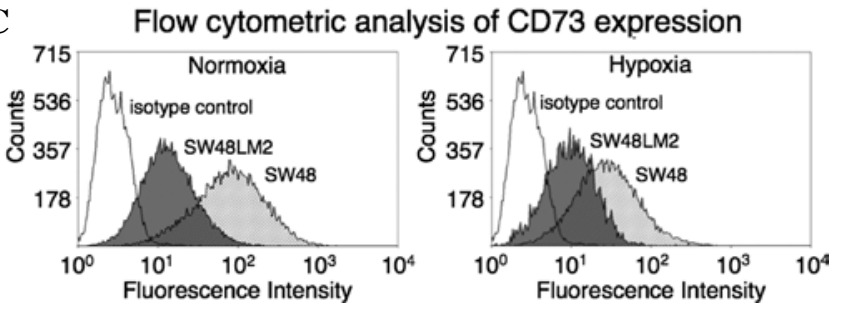

Figure 4. (A) The profiles of nucleotide metabolites in SW48 and SW48LM2 cells cultured under normoxia and hypoxia. In SW48LM2 cells cultured under both normoxia and hypoxia, purine nucleosides (adenosine, guanosine and inosine) were significantly decreased, while purine nucleotides (AMP, GMP and IMP) were reciprocally increased. There were no significant differences in the amounts of pyrimidine nucleosides (thymidine, cytidine and uridine) or nucleotides (TMP, CMP and UMP) between SW48 and SW48LM2 cells. (B) Gene expression profiles of purine nucleotidases other than CD73 in SW48 and SW48LM2 cells cultured under normoxia and hypoxia. Gene expression of NT5C2 and NT5M was not significantly different between the two cell lines. SW48n, LM2n, SW48h and LM2h indicate SW48 cells under normoxia, SW48LM2 cells under normoxia, SW48 cells under hypoxia and SW48LM2 cells under hypoxia, respectively. (C) Flow cytometric analysis of CD73 expression in SW48 and SW48LM2 cells. Representative results of three or more experiments are shown.

differences were noted in the expression levels of either the NT5M or NT5C2 gene between the SW48 and SW48LM2 cells cultured under normoxia and hypoxia (Fig 4B). Gene transcript levels of NT5CA1 and NT5CB1 were undetectable within the evaluable cycle numbers of real-time RT-PCR. The flow cytometric analysis showed an evident reduction in CD73 expression in the SW48LM2 cells (Figs. 3 and 4C). Purine nucleosides generated extracellularly by CD73 can be transferred into tumor cells via their nucleoside transporters, such as ENT1 and ENT2 (17). Collectively, decreased purine nucleosides and reciprocally increased purine nucleotides in the SW48LM2 cells appeared to be associated with a reduced CD73 expression.

\section{Discussion}

It was previously reported that tumor cells metastasizing to lymph nodes highly express CD73 $(9,10)$. As adenosine generated by CD73 restricts lymphocyte migration, it is plausible that CD73 expression in tumor cells leads to prevention against lymphocyte attacks (18). On the other hand, in the liver metastasis model using NOG mice, hepatic resident macrophages likely play a role in the first line of defense against circulating tumor cells in the absence of T, B and NK cells. Adenosine enhances macrophages to produce nitric oxide (NO) (19). The reduction in CD73 expression in tumor cells may negatively control hepatic tumoricidal macrophages via the loss of effects of adenosine on NO production. Experimental efforts are underway to functionally examine whether such mechanisms result in SW48LM2 cells being highly metastatic to the liver.

As suggested by the morphological differences in the spheroid formation between SW48 and SW48LM2 cells, potential mechanisms other than the negative control of macrophages should also be investigated to explain the characteristics of SW48LM2 cells. It is possible that adenosine extracellularly generated by CD73 in tumor cells influences, not only adjacent non-tumor cells, but also the tumor cells themselves in an autocrine and/or paracrine manner (17). In a previous study, the chemical inhibition of CD73 enzymic activities led to the decrease in both proliferation and apoptosis of breast cancer cells (20). Similarly, a reduction in CD73 expression may not only slow cell cycling, but also lead to resistance to apoptosis, potentially contributing to tumor progression.

In addition to CD73, it is important to ascertain whether other molecules, highly or poorly expressed, are also functionally associated with the distinct features of SW48LM2 cells. The increased expression of ALDH1L2, ALDH3A1 and VEGF189 by SW48LM2 cells appears to be compatible with previous studies which found a positive association of their expression with tumor growth and/or metastases $(14,15)$. In contrast, a decreased expression of the S100A4 and MCT4 genes conflicts with previous findings suggesting that there is a positive association of their expression with tumor progression $(2,11,12)$. In particular, S100A4 has been identified as a key molecular regulator of liver metastases through the establishment of a highly metastatic pancreatic cancer cell subline in a manner similar to the present study (2). Our results highlight the need for interpretative caution when investigating metastasis-associated molecules for diagnostic and therapeutic applications and provide insights into the mechanisms of liver metastases. 


\section{Acknowledgements}

The present study was supported, in part, by the Keio University Global Center of Excellence (G-COE) Program 'Center for Human Metabolomic Systems Biology' funded by the Ministry of Education, Culture, Sport, Science and Technology, Japan, as well as the Prototype Validation/ Practical Realization Program for Advanced Measurement and Analysis (Program-P) funded by the Japan Science and Technology Agency, Japan. We thank Dr Toshihide Imaizumi for his consideration in completing the present study. We also thank Ms. Yoshiko Nagahata and Ms. Tomomi Matsuura for the help in running CE-MS.

\section{References}

1. Ito M, Hiramatsu H, Kobayashi K, Suzue K , Kawahata M, Hioki K, Ueyama Y, Koyanagi Y, Sugamura K, Tsuji K, Heike T and Nakahata T: NOD/SCID/gamma(c)(null) mouse: an excellent recipient mouse model for engraftment of human cells. Blood 100: 3175-3182, 2002.

2. Suemizu H, Monnai M, Ohnishi Y, Ito M, Tamaoki N and Nakamura M: Identification of a key molecular regulator of liver metastasis in human pancreatic carcinoma using a novel quantitative model of metastasis in NOD/SCID/ $\gamma$ cnull (NOG) mice. Int J Oncol 31: 741-751, 2007.

3. Ruers $\mathrm{T}$ and Bleichrodt RP: Treatment of liver metastases, an update on the possibilities and results. Eur J Cancer 38: 1023-1033, 2002.

4. Khatib AM, Fallavollita L, Wancewicz EV, Monia BP and Brodt P: Inhibition of hepatic endothelial E-selectin expression by C-raf antisense oligonucleotides blocks colorectal carcinoma liver metastasis. Cancer Res 62: 5393-5398, 2002.

5. Hamada K, Monnai M, Kawai K, Nishime C, Miyazaki N, Ohnishi Y, Nakamura M and Suemizu H: Liver metastasis models of colon cancer for evaluation of drug efficacy using NOD/Shi-scid IL2R $\gamma$ null (NOG) mice. Int J Oncol 32: 153-159, 2008 .

6. Abu-Eid R and Landini G: Morphometrical differences between pseudo-epitheliomatous hyperplasia in granular cell tumors and squamous cell carcinomas. Histopathology 48: 407-416, 2006.

7. Soga T, Baran R, Suematsu M, Ueno Y, Ikeda S, Sakurakawa T, Kakazu Y, Ishikawa T, Robert M, Nishioka T and Tomita M: Differential metabolomics reveals ophthalmic acid as an oxidative stress biomarker indicating hepatic glutathione consumption. J Biol Chem 281: 16768-16776, 2006.

8. Frieboes HB, Zheng X, Sun CH, Tromberg B, Gatenby R and Cristini V: An integrated computational/experimental model of tumor invasion. Cancer Res 66: 1597-1604, 2006.
9. Lee H, Lin EC, Liu L and Smith JW: Gene expression profiling of tumor xenografts: in vivo analysis of organ-specific metastasis. Int J Cancer 107: 528-534, 2003.

10. Leth-Larsen R, Lund R, Hansen HV, Laenkholm AV, Tarin D, Jensen ON and Ditzel HJ: Metastasis-related plasma membrane proteins of human breast cancer cells identified by comparative quantitative mass spectrometry. Mol Cell Proteomics 8: 1436-1449, 2009.

11. Gallagher SM, Castorino JJ, Wang D and Philp NJ: Monocarboxylate transporter 4 regulates maturation and trafficking of CD147 to the plasma membrane in the metastatic breast cancer cell line MDA-MB-231. Cancer Res 67: 4182-4189, 2007.

12. Saleem M, Kweon MH, Johnson JJ, Adhami VM, Elcheva I, Khan N, Bin Hafeez B, Bhat KM, Sarfaraz S, Reagan-Shaw S, Spiegelman VS, Setaluri V and Mukhtar H: S100A4 accelerates tumorigenesis and invasion of human prostate cancer through the transcriptional regulation of matrix metalloproteinase 9. Proc Natl Acad Sci USA 103: 14825-14830, 2006.

13. Salaun B, Coste I, Rissoan MC, Lebecque SJ and Renno T: TLR3 can directly trigger apoptosis in human cancer cells. J Immunol 176: 4894-4901, 2006.

14. Moreb JS, Baker HV, Chang LJ, Amaya M, Lopez MC, Ostmark B and Chou W: ALDH isozymes downregulation affects cell growth, cell motility and gene expression in lung cancer cells. Mol Cancer 7: 87, 2008.

15. Tokunaga T, Oshika Y, Abe Y, Ozeki Y, Sadahiro S, Kijima H, Tsuchida T, Yamazaki H, Ueyama Y, Tamaoki $\mathrm{N}$ and Nakamura M: Vascular endothelial growth factor (VEGF) mRNA isoform expression pattern is correlated with liver metastasis and poor prognosis in colon cancer. Br J Cancer 77: 998-1002, 1998.

16. Brabletz T, Jung A, Spaderna S, Hlubek F and Kirchner T: Opinion: migrating cancer stem cells - an integrated concept of malignant tumour progression. Nat Rev Cancer 5: 744-749, 2005.

17. Cho SY, Polster L, Engles JM, Hilton J, Abraham EH and Wahl RL: In vitro evaluation of adenosine 5'-monophosphate as an imaging agent of tumor metabolism. J Nucl Med 47: 837-845, 2006.

18. Takedachi M, Qu D, Ebisuno Y, Oohara H, Joachims ML, McGee ST, Maeda E, McEver RP, Tanaka T, Miyasaka M, Murakami S, Krahn T, Blackbum MR and Thompson LF: CD73-generated adenosine restricts lymphocyte migration into draining lymph nodes. J Immunol 180: 6288-6296, 2008.

19. Hasko G, Szabo C, Nemeth ZH, Kvetan V, Pastores SM and Vizi ES: Adenosine receptor agonists differentially regulate IL-10, TNF- $\alpha$ and nitric oxide production in RAW 264.7 macrophages and in endotoxemic mice. J Immunol 157: 4634-4640, 1996.

20. Zhou X, Zhi X, Zhou P, Chen S, Zhao F, Shao Z, Ou Z and Yin L: Effects of ecto-5'-nucleotidase on human breast cancer cell growth in vitro and in vivo. Oncol Rep 17: 1341-1346, 2007. 